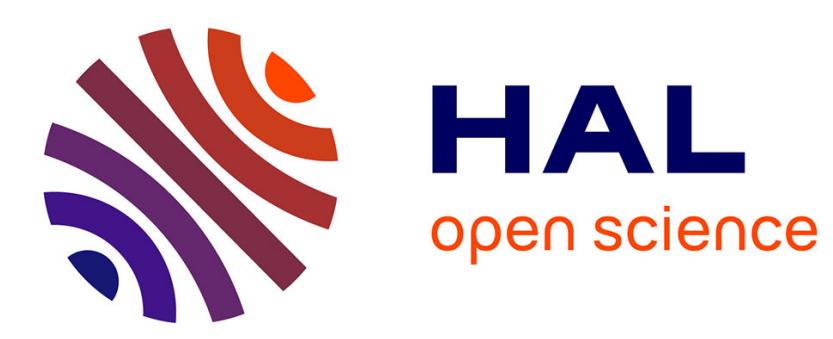

\title{
About the stability of nonconservative undamped elastic systems:some new elements
}

\author{
Jean Lerbet, Elie Absi, Alain Rigolot
}

\section{To cite this version:}

Jean Lerbet, Elie Absi, Alain Rigolot. About the stability of nonconservative undamped elastic systems:some new elements. International Journal of Structural Stability and Dynamics, 2009, 9 (2), pp.357-367. 10.1142/S0219455409003065 . hal-00342816

\section{HAL Id: hal-00342816 https://hal.science/hal-00342816}

Submitted on 26 Dec 2019

HAL is a multi-disciplinary open access archive for the deposit and dissemination of scientific research documents, whether they are published or not. The documents may come from teaching and research institutions in France or abroad, or from public or private research centers.
L'archive ouverte pluridisciplinaire HAL, est destinée au dépôt et à la diffusion de documents scientifiques de niveau recherche, publiés ou non, émanant des établissements d'enseignement et de recherche français ou étrangers, des laboratoires publics ou privés. 


\title{
ABOUT THE STABILITY OF NONCONSERVATIVE UNDAMPED ELASTIC SYSTEMS: SOME NEW ELEMENTS
}

\author{
JEAN LERBET \\ IBISC, Université d'Evry Val d'Essone, 40 Rue Pelvoux \\ CE 1455 Courcouronnes, 91020 Evry Cedex, France \\ jean.lerbet@ibisc.univ-evry.fr \\ ELIE ABSI \\ Professeur honoraire à l'Ecole Centrale de Paris \\ 44 Rue de Cronstadt, 75015 Paris, France \\ ALAIN RIGOLOT \\ LMM, Paris VI \\ 4 Place Jussieu, 75005 Paris, France
}

Received 29 July 2008

Accepted 28 August 2008

\begin{abstract}
It is well-known that the domains of static stability and dynamic stability (even for a linear approach) do not match each other when the system is no more conservative and the dynamic approach is usually privileged, meaning that the dynamic stability domain is included in the static one. Following previous works proposing a new criterion of static stability of nonconservative systems and prolonging a paper of Gallina devoted to linear dynamic instability (flutter), we show in this paper some remarkable relations between the two approaches: contrary to the common thought, the new static stability criterion implies partially the dynamic one.
\end{abstract}

Keywords: Nonconservative systems; static stability; dynamic stability; stiffness matrix; mixed perturbation.

\section{Introduction}

In Ref. 1, Gallina introduces new algebra and symbolic computation results ${ }^{2}$ for the study of nonconservative undamped elastic systems. These results are interesting because they provide the critical load of dynamic instability (flutter) for such systems. Unfortunately, the algebraic conditions do not seem to have any mechanical basis. The aim of this paper is to make these formal conditions meaningful and to draw conclusions about comparison between static and dynamic stabilities from these results.

Gallina splits his result into two theorems. The first one deals with the resultant of the system's characteristic polynome $P$ and its derivative $P^{\prime}$, the second with 
the sign of the coefficients of $P$. Our paper addresses the second theorem, for which we provide a mechanical basis.

As in a previous paper, ${ }^{3}$ we shall first give a new definition of static instability. Such a definition coincides with the classical one (divergence) for conservative systems but is better adapted to nonconservative systems. This definition leads to a new criterion of static stability.

Some of the properties of this criterion are then introduced, and finally the criterion is applied to the stability conditions given by the second theorem in Ref. 1 and one can then demonstrate that they are automatically satisfied, thus providing the mechanical basis for the conditions of the second theorem.

Following other papers ${ }^{3,4}$ where examples illustrate this fact, such a result also suggests that the stability of structures should be investigated through both static and dynamic approaches. Contrary to the common opinion privileging systematically the dynamic approach, the better criterion for ensuring stability should be chosen in each case after both calculations. An example coming from aerodynamics and another about Ziegler's model submitted to a partial follower force illustrate finally our approach and our result.

Before starting the development of the paper, let us note that the background of this work is the linear theory.

\section{A New Definition of Static Stability}

\subsection{Stability definitions and conservative systems}

We shall first investigate the stability of the equilibrium in a discrete mechanical system represented by 0 . It is assumed that this system is nonconservative but elastic. The linearized equations of the system become

$$
\mathbf{M} \delta \ddot{\mathbf{x}}+\mathbf{K}(\lambda) \delta \mathbf{x}=0,
$$

where $\delta \mathbf{x}=\operatorname{col}\left(\delta x_{1}, \ldots, \delta x_{n}\right)$ is an unknown vector describing the deformation of the system, $\mathbf{M}$ the mass matrix, $\mathbf{K}=\mathbf{K}(\lambda)$ the stiffness matrix and $\lambda$ the loading parameter. $\mathbf{M}$ is symmetric positive definite and $\mathbf{K}(\lambda)$ is any matrix that in a conservative system is symmetric positive. Before we detail the new approach, the classical approach in statics and dynamics is now recalled.

More precisely, there is a function $F:(\lambda, q) \mapsto F(\lambda, q)$ such that $q=0$ is a solution to the complete equilibrium equations,

$$
F(\lambda, q)=0 \quad \forall \lambda \in[0, \alpha],
$$

and then $\frac{\partial F}{\partial q}(\lambda, 0)=\mathbf{K}(\lambda)$. The equilibrium of the system is said to be stable or dynamically stable when for minute initial conditions of position and velocity, $\|q(0)\| \leq \epsilon,\|\dot{q}(0)\| \leq \epsilon$, the solution $t \mapsto \mathbf{q}(t)=\left(q_{1}(t), \ldots, q_{n}(t)\right)$ of the nonlinearized dynamic equations remains in a small well-defined neighborhood around the origin $\|\mathbf{q}(t)\| \leq O(\epsilon) \forall t>0$. The main difficulty, not studied here, is to conclude about the stability using only linearized equations. If only the solution of the 
linearized equations is stable in the same sense as for the nonlinearized equations, one speaks of weak stability. In the rest of the paper, we shall only look at weak stability.

Static instability, i.e. the problem of the bifurcation, concerns the study of the solutions $t \mapsto \mathbf{q}(t)$ to Eq. (2). The associated linearized problem is

$$
\mathbf{K}(\lambda) \delta \mathbf{x}=\mathbf{0} .
$$

Equation (3) may be interpreted as the homogeneous problem associated with the nonhomogeneous one formulated by Absi..$^{5,6}$

A system is considered statically stable if for any infinitesimal perturbation $\delta \mathbf{s}$ of the actions, the vector of the coordinates of generalized effective actions, the system moves infinitesimally to a new equilibrium defined by $\delta \mathbf{x} . \delta \mathbf{x}$ and $\delta \mathbf{s}$ follow the equations

$$
\delta \mathbf{s}=\mathbf{K}(\lambda) \delta \mathbf{x} .
$$

In the following, $\mathbf{K}(0)$ is regular and symmetric (elastic system) and we are looking for a value of $\lambda>0$ (the lowest) for which stability is not ensured. It is called a critical value of loading and:

(a) In classical static instability or divergence [in the case of Eq. (3)], we shall speak of $s$ instability and $\lambda_{s}$ is its corresponding value.

(b) In dynamic instability (in the case of complete dynamic equations), we shall speak of $d$ instability and $\lambda_{d}$ is its corresponding value.

(c) In weak dynamic instability or flutter [in the case of Eq. (1)], we shall speak of $w$ instability and $\lambda_{w}$ is its corresponding value.

It is known that, for a conservative system, $s$ instability and $w$ instability appear for the same value of the parameter of loading $\lambda_{s}=\lambda_{w}$ obtained by the equation

$$
\operatorname{det}(\mathbf{K}(\lambda))=0 .
$$

Moreover, the (complete) stability is ensured by an energy criterion preventing movement in the system if it is stable $\left(\lambda_{s}=\lambda_{w}=\lambda_{d}\right)$.

\subsection{Mixed perturbation: a new stability criterion}

We propose a new formulation of static stability which makes, use of the concept of mixed perturbation. Because of the symmetry of the matrix $\mathbf{K}$ in a conservative system, the variations $\delta \mathbf{s}$ and $\delta \mathbf{x}$ mentioned above play a symmetric role and only one pure perturbation, $\delta \mathbf{s}$, is needed to analyze static stability. However, in nonconservative systems, the nonsymmetry of $\mathbf{K}$ implies that the stability has to be studied together with the so-called system's mixed perturbations. This means that we need to consider in Eq. (4) perturbations like $\left(\delta s_{i_{1}}, \ldots, \delta s_{i_{k}}, \delta x_{i_{k+1}}, \ldots, \delta x_{i_{n}}\right)$ for all $i_{1}, \ldots i_{k}$ because of the duality between the displacements $\delta \mathbf{x}$ and the external actions $\delta$ s. 
Definition. The system is said to be statically stable if the linear system

$$
\delta \mathbf{s}=\mathbf{K}(\lambda) \delta \mathbf{x}
$$

is nonsingular for all mixed perturbation $\left(\delta s_{i_{1}}, \ldots, \delta s_{i_{k}}, \delta x_{i_{k+1}}, \ldots, \delta x_{i_{n}}\right)$. As in reallife, it is also assumed that $(\delta \mathbf{s} \mid \delta \mathbf{x}) \geq 0$ for any mixed perturbation. This criterion is obviously the direct generalization of the stability criterion of conservative systems.

Notation. In the new static criterion of instability, we shall speak of $m$ instability and $\lambda_{m}$ is its corresponding value.

Therefore, $m$ instability appears when $\mathbf{K}(\lambda)$ is no longer positive definite or if there is a vector $\mathbf{x} \neq \mathbf{0}$ such that ${ }^{t} \mathbf{x} \mathbf{K} \mathbf{x} \leq 0$. More precisely, $\mathbf{K}=\mathbf{K}(\lambda)$ and $\mathbf{K}(0)$ is symmetric positive definite (a conservative system if $\lambda=0$ ). Consequently, by continuity, the system stops being $m$ stable if $\mathbf{K}(\lambda)$ stops being positive definite or, in this study, if there is a vector $\mathbf{x} \neq \mathbf{0}$ such that ${ }^{t} \mathbf{x K}(\lambda) \mathbf{x}=0$.

It is usual to speak of positive definite symmetric matrices. But the property of positive definiteness may be defined for any matrix by:

Definition. Let $\mathbf{A}$ be a square matrix. A is said to be positive definite if and only if

$$
{ }^{t} \mathbf{x} \mathbf{A} \mathbf{x}>0 \quad \forall \mathbf{x} \in \mathbb{R}^{n} \mathbf{x} \neq \mathbf{0} .
$$

The proposed new criterion leads us to analyze these positive definite matrices (symmetric or not).

\section{About Positive Definite Matrices}

If $\mathbf{A}$ is a matrix, $\mathbf{A}_{s}=\frac{\mathbf{A}+{ }^{t} \mathbf{A}}{2}$ and $\mathbf{A}_{a}=\frac{\mathbf{A}-{ }^{t} \mathbf{A}}{2}$ are respectively the symmetric and the skew-symmetric parts of $\mathbf{A}$. It is clear that for all $\mathbf{x}$ in $\mathbb{R}^{n}$,

$$
{ }^{t} \mathbf{x} \mathbf{A x}={ }^{t} \mathbf{x} \mathbf{A}_{s} \mathbf{x}
$$

The $m$ criterion concerns only the symmetric part of the system's stiffness matrix and there is no way we can get direct relationships between the proper values of $\mathbf{A}$ and $\mathbf{A}_{s}$.

However, some specific properties of a positive definite matrix, whether it is symmetric or not, remain valid. It is known that for a symmetric matrix $\mathbf{A}, \mathbf{A}$ is positive definite if and only if all principal minors are positive [a principal minor $d_{i}(\mathbf{A})$ is the determinant of the matrix obtained by suppressing row $i$ and column $i$. Positive definite matrices follow Theorem 1:

Theorem 1. If $\mathbf{A}$ is a positive definite matrix, then every principal minor of $\mathbf{A}$ is positive.

Before this is demonstrated, we can note that a positive definite matrix has the following property: If $\mathbf{A}$ is a positive definite matrix, every principal submatrix is also positive definite. Consequently, any submatrix $s(\mathbf{A})$ obtained by suppressing the same lines and columns is positive definite too. 
Proof. Let $k$ be the suppressed line and column index of $\mathbf{A}$, resulting in matrix $s(\mathbf{A})$, and $\mathbf{y} \neq 0$ in $\mathbb{R}^{n-1}$. In addition, let $\mathbf{x} \neq 0$ be in $\mathbb{R}^{n}$ such that $x_{k}=0, x_{i}=y_{i}$ if $i<k$ and $x_{i}=y_{i-1}$ if $i>k$. The condition ${ }^{t} \mathbf{x A x}>0$ becomes ${ }^{t} \mathbf{y} s(\mathbf{A}) \mathbf{y}>0$ and $s(\mathbf{A})$ is positive definite.

Proof of Theorem 1. According to the previous paragraph, it is enough to prove that the determinant of $\mathbf{A}$ is strictly positive if $\mathbf{A}$ is positive definite. Decomposing $\mathbf{A}=\mathbf{A}_{s}+\mathbf{A}_{a}$ and noting that $\mathbf{A}_{s}$ is symmetric positive definite, let us consider $\mathbf{B}$ the square root of $\mathbf{A}_{s}$ and let us write

$$
\mathbf{A}={ }^{t} \mathbf{B}\left(\mathbf{I}+{ }^{t} \mathbf{B}^{-1} \mathbf{A}_{a} \mathbf{B}^{-1}\right) \mathbf{B}={ }^{t} \mathbf{B}\left(\mathbf{I}+\mathbf{A}_{m}\right) \mathbf{B} .
$$

$\mathbf{A}_{m}$ is skew-symmetric and

$$
\operatorname{det}(\mathbf{A})=\operatorname{det}\left({ }^{t} \mathbf{B B}\right) \operatorname{det}\left(\mathbf{I}+\mathbf{A}_{m}\right)=\operatorname{det}\left(\mathbf{A}_{s}\right) \operatorname{det}\left(\mathbf{I}+\mathbf{A}_{m}\right)>0
$$

if and only if $\operatorname{det}\left(\mathbf{I}+\mathbf{A}_{m}\right)>0$ (because $\operatorname{det}\left(\mathbf{A}_{s}\right)>0$ ).

The properties of a skew-symmetric matrix depend on the dimension $n$ of the space, i.e. of the size of the matrix (see Ref. 7, for example): if $n=2 p+1$ ( $n$ is odd), $\mathbf{A}_{m}$ is singular. Let $u_{m}$ be the morphism canonically attached to $\mathbf{A}_{m}$ and $E=\operatorname{Ker}\left(u_{m}\right)$.

Because $u_{m}(\mathbf{x} \mid \mathbf{y})=-\left(\mathbf{x} \mid u_{m}(\mathbf{y})\right)$ for all $\mathbf{x}$ and $\mathbf{y}, E$ and $E^{\perp}$ are $u_{m}$-stable. Considering the restriction $v_{m}$ of $u_{m}$ to $E^{\perp}, v_{m}$ is skew-symmetric and nonsingular and $\operatorname{dim}\left(E^{\perp}\right)=2 p$. There is a basis in which the matrix of $v_{m}$ is $\operatorname{diag}\left(\mathbf{Q}_{1}, \ldots, \mathbf{Q}_{p}\right)$ with $\mathbf{Q}_{k}=q_{k} \mathbf{S}$ and

$$
\mathbf{S}=\left(\begin{array}{rr}
0 & -1 \\
1 & 0
\end{array}\right)
$$

Proof. $v_{m}^{2}$ is symmetric real and if $\lambda$ is a proper value of $v_{m}^{2}$ and $\mathbf{x}$ a proper vector, then $v_{m}(\mathbf{x})$ is also a proper vector. There are $\lambda_{1}, \ldots, \lambda_{p}$ proper values with $\mathbf{x}_{1}, v_{m}\left(\mathbf{x}_{1}\right), \ldots, \mathbf{x}_{p}, v_{m}\left(\mathbf{x}_{p}\right)$ associated proper vectors. By normalizing these vectors, the required basis is then obtained.]

It follows that

$$
\operatorname{det}\left(\mathbf{I}+\mathbf{A}_{m}\right)=\prod_{k=1}^{n}\left(1+q_{k}^{2}\right)>0 .
$$

Remark. Another proof of this result may be directly obtained by using the following result (see Ref. 9): If $S$ is symmetric positive definite and $A$ anti-symmetric then $\operatorname{det}(S+A) \geq \operatorname{det}(S)$. By applying this result to the previous decomposition $\mathbf{A}=\mathbf{A}_{s}+\mathbf{A}_{a}$ and to apply standard results (Sylvester's theorem) concerning symmetric definite positive matrices, one get another proof of Theorem 1 .

\section{Application to the Stability of Nonconservative Undamped Systems}

Let $\Sigma$ be a nonconservative undamped system, and $\mathbf{M}$ and $\mathbf{K}$ its mass and stiffness matrices. In addition, let $P(\lambda)=\operatorname{det}(\mathbf{M} \lambda+\mathbf{K})$ be its characteristic polynomial. 
The following property holds:

Theorem 2. If $\Sigma$ is $m$ stable, then the coefficients of $P(\lambda)$ are positive.

Proof. According to the definition of the $m$ stability, $\mathbf{M}$ is symmetric positive definite and $\mathbf{K}$ is positive definite. Let $\mathbf{A}$ be the square root of $\mathbf{M}$; then $\mathbf{A}$ is also symmetric positive definite, ${ }^{t} \mathbf{A} \mathbf{A}=\mathbf{M}$ and

$$
\mathbf{M} \lambda+\mathbf{K}={ }^{t} \mathbf{A}\left(\lambda \mathbf{I}+{ }^{t} \mathbf{A}^{-1} \mathbf{K} \mathbf{A}^{-1}\right) \mathbf{A}={ }^{t} \mathbf{A}\left(\lambda \mathbf{I}+\mathbf{K}_{A}\right) \mathbf{A},
$$

with $\mathbf{K}_{A}={ }^{t} \mathbf{A}^{-1} \mathbf{K A}^{-1}$. Note that because $\mathbf{A}^{-1}$ is symmetric positive definite, $\mathbf{K}_{A}$ is positive definite like $\mathbf{K}$ itself ( ${ }^{t} \mathbf{x} \mathbf{K}_{A} \mathbf{x}={ }^{t} \mathbf{y} \mathbf{K} \mathbf{y}$ with $\mathbf{y}=\mathbf{A}^{-1} \mathbf{x}$ and $\mathbf{x} \mapsto \mathbf{A}^{-1} \mathbf{x}$ is an automorphism of $\mathbb{R}^{n}$ ).

We deduce that

$$
P(\lambda)=\operatorname{det}(\mathbf{M}) \operatorname{det}\left(\lambda \mathbf{I}+\mathbf{K}_{A}\right) .
$$

If we now consider the polynomial $\mathbf{K}_{A}=\left(r_{i j}\right)_{1 \leq i, j \leq n}$,

$$
Q\left(X_{1}, \ldots, X_{n}\right)=\operatorname{det}\left(\begin{array}{cccc}
X_{1}+r_{11} & r_{12} & \ldots & r_{1 n} \\
r_{21} & X_{2}+r_{22} & \ldots & r_{2 n} \\
\vdots & & & \vdots \\
r_{n 1} & r_{n 2} & \ldots & X_{n}+r_{n n}
\end{array}\right) .
$$

Then $P(\lambda)=Q(\lambda, \ldots, \lambda)$ and

$$
Q\left(X_{1}, \ldots, X_{n}\right)=\sum_{p} \sum_{i_{1}<\cdots<i_{p}} X_{i_{1}}, \ldots, X_{i_{p}} \operatorname{det}\left(s_{i_{1}, \ldots, i_{p}}\left(\mathbf{K}_{A}\right)\right),
$$

where $s_{i_{1}, \ldots, i_{p}}\left(\mathbf{K}_{A}\right)$ is the submatrix of $\mathbf{K}_{A}$ obtained by suppression of lines and columns $i_{1}, \ldots, i_{p}$. According to Theorem 1, the coefficients of $Q$ and consequently of $P$ are positive.

\section{Examples}

Two examples are investigated. The first one is issued from aerodynamics and is a two degree of freedom model of a wing. The second one is the Ziegler' system under partial follower force.

\subsection{A two dof wing}

The chosen example comes from aerodynamics. This example may be found in Ref. 10. The system is a two degrees of freedom model of a wing (see Fig. 1). The loading parameter $\lambda$ is the speed $V$ of the wind. Let be $K_{1}$ and $K_{2}$ the bending and torsion stiffness of the system, $\theta$ the bending angle and $\psi$ the torsion angle. We investigate the stability of relatif equilibrium of the wing compared to the plane. In equilibrium position, the wing is supposed plane in the plan $O X Z$, the speed $V$ 


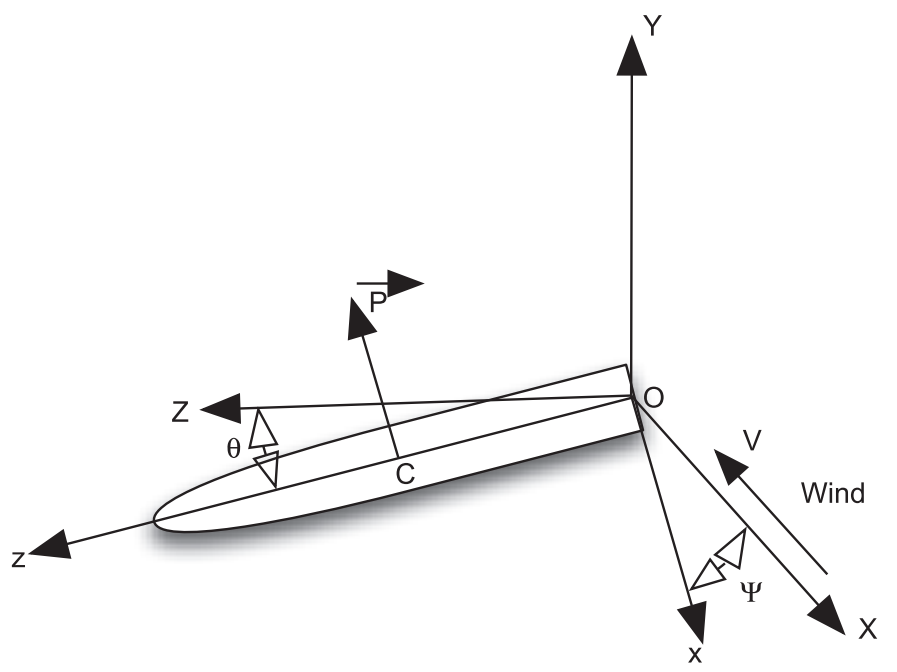

Fig. 1. Example 1.

of the wing is opposed to axis $O X$ and is constant. The frame $0 x y z$ is supposed attached to the wing that is regarded as a rigid body and $O x$ lies in the plan $O X Y$. The aerodynamic actions are reduced to a drag balanced by the reactions of the plane on the wing and to a lift $\vec{L}$ perpendicular to the wing regarded as a plate. This force is applied at the point $C$ on the axis $O z$ such as $O C=a$. The intensity $L$ of the lift force is then given by $L=\Gamma S V^{2} \psi$ where $\Gamma$ is a shape coefficient and $S$ is the surface of the wing. Straightforward calculations lead to both following expressions:

the mass matrix is given by

$$
\mathbf{M}=\left[\begin{array}{cc}
A & -E \\
-E & C
\end{array}\right]
$$

and the stiffness matrix is

$$
\mathbf{K}=\left[\begin{array}{cc}
K_{1} & a \Gamma S V^{2} \\
0 & K_{2}
\end{array}\right]
$$

We now investigate the critical values of $V$ according to the different approaches of stability and the eventual relations between themselves.

\subsubsection{Classical static instability or divergence}

$\operatorname{det}(K(V))=K_{1} K_{2}$. There is no critical value of divergence (S-instability) of the speed wind!!: $U_{s}=+\infty$. 


\subsubsection{Weak instability or Flutter}

The characteristic polynomial of the system is:

$$
P(x)=(1-\alpha) x^{2}+\left(\omega_{1}^{2}+\omega_{2}^{2}+\frac{\alpha \Gamma a S V^{2}}{E}\right) x+\omega_{1}^{2} \omega_{2}^{2}
$$

where $0 \leq \alpha=\frac{E^{2}}{A C}<1$ (because $M$ is symmetric positive definite), $\omega_{1}^{2}=\frac{K_{1}}{A}$, $\omega_{2}^{2}=\frac{K_{2}}{C}$. Straightforward calculations give:

$$
\Delta=\left(\omega_{1}-\omega_{2}\right)^{2}+4 \alpha \omega_{1}^{2} \omega_{2}^{2}+\frac{\alpha \Gamma a S V^{2}}{E}\left(\frac{\alpha \Gamma a S V^{2}}{E}+2\left(\omega_{1}^{2}+\omega_{2}^{2}\right)\right)
$$

- If $E>0$ there is no critical value of flutter of the speed of wind!!: $U_{w}=+\infty$.

- If $E<0$ the critical value $U_{w}$ of flutter ( $w$ instability) is then:

$$
U_{w}^{2}=-\frac{E}{\alpha a \Gamma S}\left(\omega_{1}^{2}+\omega_{2}^{2}-2 \omega_{1} \omega_{2} \sqrt{1-\alpha}\right)
$$

obtaining by annulating $\Delta$ according to Gallina's criterion because if $V<U_{w}$ the coefficients of $P$ are $>0$. Indeed, $V<U_{w}$ implies $\omega_{1}^{2}+\omega_{2}^{2}+\frac{\alpha \Gamma a S V^{2}}{E}>0$, the other coefficients of $P$ being obviously positive.

\subsubsection{New criterion of static stabilty}

Calculations give here the following expression of critical value $U_{m}$ of static instability according to the new criterion ( $m$ instability):

$$
U_{m}^{2}=\frac{2 \sqrt{K_{1} K_{2}}}{\Gamma a S}=\frac{2 \omega_{1} \omega_{2} \sqrt{A C}}{\Gamma a S}= \pm \frac{2 \omega_{1} \omega_{2} E}{\Gamma a S \sqrt{\alpha}}
$$

according to the sign of $E$.

We first may note that there always is a critical speed $U_{m}$ of wind (for $E>0$ or for $E<0$ ) and obviously:

- $U_{m}<U_{s}=+\infty$ (see Theorem 1$)$,

- for $E>0$ then $U_{m}<U_{w}=+\infty$,

- for $E<0$,

$$
\frac{U_{w}^{2}}{U_{m}^{2}}=\frac{1}{2 \sqrt{\alpha}}\left(\frac{\omega_{1}}{\omega_{2}}+\frac{\omega_{2}}{\omega_{1}}-2 \sqrt{1-\alpha}\right)
$$

that may be $>1$ or $<1$ which means the comparison between the both criteria depends on the mass repartition $(A, C, E)$ of the structure.

To conclude with this example, let us observe how it works concerning the Theorem 2.

If $V<U_{m}$, then:

$$
\begin{aligned}
\omega_{1}^{2}+\omega_{2}^{2}+\frac{\alpha \Gamma a S V^{2}}{E} & =\omega_{1}^{2}+\omega_{2}^{2}+2 \omega_{1} \omega_{2} \frac{V^{2}}{U_{m}^{2}} \frac{E}{\sqrt{A C}} \\
& >\omega_{1}^{2}+\omega_{2}^{2}-2 \omega_{1} \omega_{2}=\left(\omega_{1}-\omega_{2}\right)^{2} \geq 0
\end{aligned}
$$


because $-\sqrt{A C} \leq E \leq \sqrt{A C}$ and $V<U_{m}$. We observe on this example the results of Theorems 1 and 2. We see also that no general implication exists between the both criteria but only the partial implication given by the Theorem 2 .

\subsection{Ziegler's system under partial follower force}

The application of the new criterion to Ziegler's system under follower force has been already done in a previous paper. ${ }^{3}$ A straightforward calculation could show that the result of last theorem holds in the corresponding example. We now illustrate our results on a most general case of similar system under a partial follower force. With the standard notations (any mass repartition), we obtain by using standard notations (see for example Ref. 8 (see Fig. 2):

$$
\mathbf{M}=\left[\begin{array}{cc}
A & -E \\
-E & C
\end{array}\right]
$$

and the stiffness matrix is

$$
\mathbf{K}=\mathbf{K}(\alpha, p)=k\left[\begin{array}{cc}
2-p & -1+(1-\alpha) p \\
-1 & 1-\alpha p
\end{array}\right]
$$

where $\lambda=p=\frac{F l}{k}$ is the load parameter of the system and $\alpha$ is the partial follower parameter. $\alpha=1$ corresponds of the conservative case and $\alpha=0$ to the complete follower load case treated by Ziegler. In Ref. 3, some calculations using the new criterion have been already done for this case.

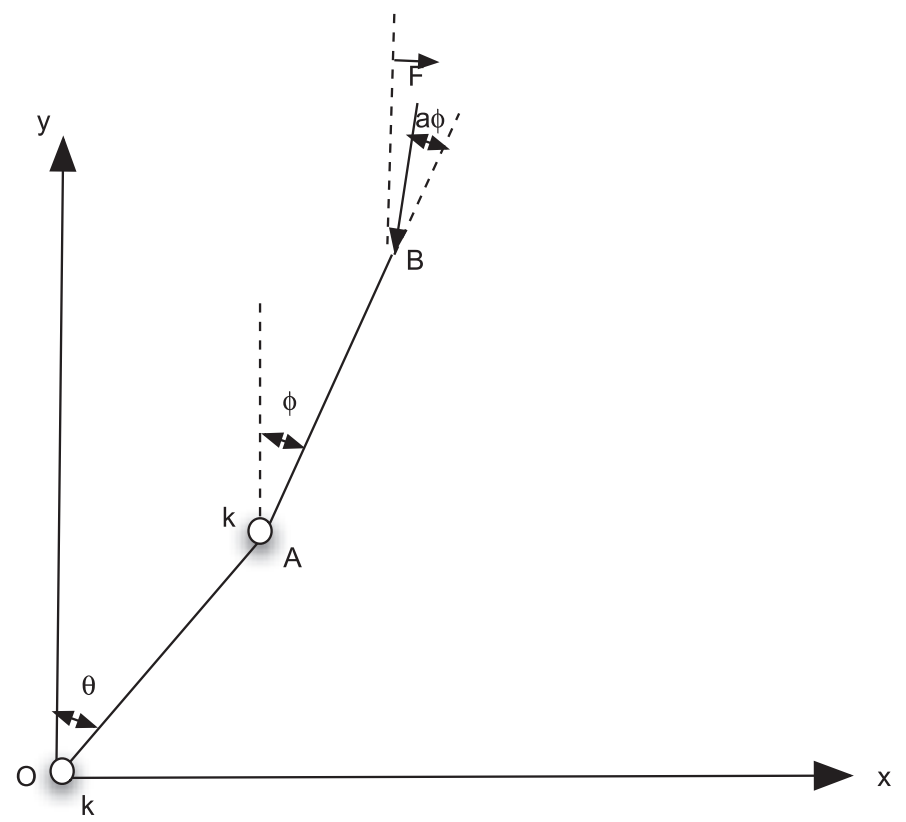

Fig. 2. Example 2. 


\subsubsection{Classical static instability or divergence}

$\operatorname{det}(K(p))=\alpha p^{2}-3 p \alpha+1=0$. That determines a region of divergence for each $\alpha$ and a curve $\alpha \mapsto p_{s}(\alpha)$.

\subsubsection{New criterion of static stability}

By applying the new criterion, we obtain a curve $\alpha \mapsto p_{m}(\alpha)$ solution of $p^{2}\left(a^{2}-\right.$ $6 a+1)+12 a p-4=0$ with $0<p<2$. On Fig. 3 , we may observe that new criterion is better than this one of divergence because of the relative position of the both curves $\alpha \mapsto p_{s}(\alpha)$ and $\alpha \mapsto p_{m}(\alpha): p_{m}(\alpha) \leq p_{s}(\alpha)$ for all $0<\alpha<1$.

\subsubsection{Weak stability}

We investigate only the case of uniform mass distribution. Calculations lead to following characteristic polynomial:

$$
P(x)=\frac{7}{4} x^{2}+\left(9-\frac{5}{2} p(1+\alpha)\right) \omega^{2} x+\omega^{4}\left(1+p^{2} \alpha-3 \alpha p\right)
$$

where $\omega^{2}=\frac{k}{C}$. Calculations show that if $p<p_{m}(\alpha)$ then $9-\frac{5}{2} p(1+\alpha)>0$ for $0<\alpha<1$ which is Theorem 2 .

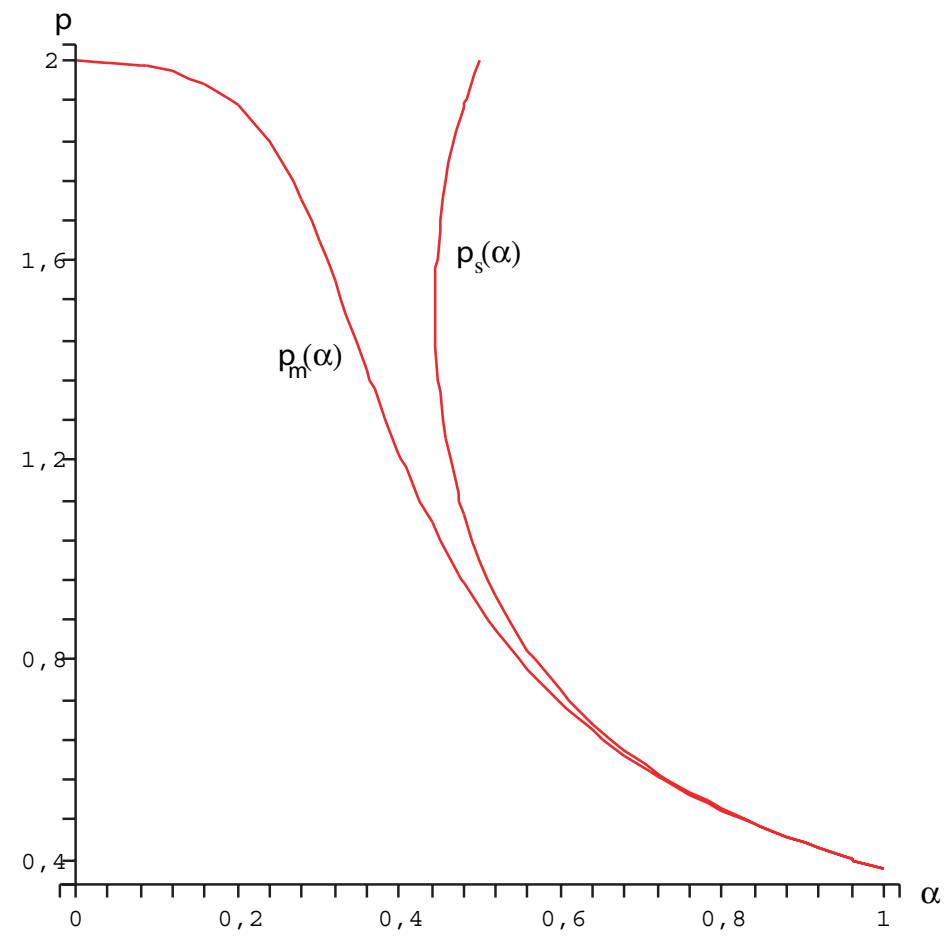

Fig. 3. Comparison of static criteria for Ziegler's system. 


\section{Conclusion}

The announced result has been demonstrated in three steps. First, a new criterion of static stability has been proposed for analyzing the static stability of any system (whether it is conservative or not). The second step consisted in analyzing the structure of positive definite operators. Thirdly, the result has been deduced: the coefficients of the characteristic polynomial of undamped nonconservative $m$ stable systems are always positive. This brings a mechanical basis to the second condition of stability in Gallina's article ${ }^{1}$ and solves it. The conditions of the first theorem should be studied in the same way.

Concerning the stability of structures, the obtained result shows that, introducing a definition of static stability more suitable for the nonconservative cases (and equivalent to the usual static stability for conservative systems), the links between the two approaches do not seem so obvious as what is usually supposed. On the contrary, the link that is only partial is in the opposite direction: if any undamped system is static $m$ stable, then the second part of conditions of its dynamic stability necessarily holds.

For engineers, this means that it would be wiser to use both approaches and to choose the safer one. Unfortunately, such a choice must be made for each structure, because there are examples where the dynamic approach leads to a lower critical load of instability and other examples where the static approach is better. ${ }^{3,4}$

The examples studied in the last section illustrate this situation.

\section{References}

1. P. Gallina, About the stability of non-conservative undamped systems, J. Sound Vib. 262 (2003) 977-988.

2. L. Yang, Recent advances on determining the number of real roots of parametric polynomials, J. Symb. Comput. 28 (1999) 225-242.

3. E. Absi and J. Lerbet, Instability of elastic bodies, Mech. Res. Commun. 31(1) (2004) 39-44.

4. E. Absi and J. Lerbet, In reply to some remarks by N. Challamel on paper "Instablity of Elastic Bodies" Mech. Res. Commun. 32 (2005) 613-615.

5. E. Absi, Instabilité des corps élastiques, Revue Française de Génie Civil 6(2) (2002) 161-196.

6. E. Absi, Critère général d'instabilité des structures, Ann. B.T.P. 1 (2002) 15-29.

7. V. N. Koshlyakov, Structural transformations of non-conservative systems, J. Appl. Math. Mech. 64(6) (2000) 895-902.

8. A. N. Kounadis, On the failure of static stability analyses of nonconservative systems in regions of divergence, Int. J. Solids Struct. 31(15) (1994) 2099-2120.

9. A. Ostrowski and O. Taussky, On the variation of the determinant of a positive definite matrix, Neder. Akadem. Wet. Proc. A54 (1951) 383-385.

10. Y. Bamberger, Mécanique de l'ingénieur (Hermann, 1981).

11. H. Ziegler, Principles of structural Stability (Blaisdell Pub. Company, 1968). 